\title{
Non-linear volatility and dynamics of the Tunisian stock market
}

\author{
Kalai Lamia ${ }^{1,2, *}$, Jilani Faouzi ${ }^{1,2}$ \\ ${ }^{1}$ Graduate Institute of Business and Accounting of Bizerta, Faculty of economic Sciences and Management of Tunisia \\ ${ }^{2}$ University of Carthage, University of Tunis El Manar \\ Email address: \\ lamiakalai@yahoo.fr (K. Lamia), faouzi.jilan@fsegt.rnu. tn (J. Faouzi) \\ To cite this article: \\ Kalai Lamia, Jilani Faouzi. Non-linear Volatility and Dynamics of the Tunisian Stock Market. International Journal of Economics, \\ Finance and Management Sciences. Vol. 2, No. 1, 2014, pp. 22-32. doi: 10.11648/j.ijefm.20140201.14
}

\begin{abstract}
Measuring and controlling risk is one of the most attractive issues in finance. With the persistence of uncontrolled and erratic stocks movements, volatility is perceived as a barometer of daily fluctuations. An objective measure of this variable seems then needed to control risks and cover those that are considered the most important. Non-linear autoregressive modeling is our first evaluation approach. In particular, we test the presence of "persistence" of conditional variance and the presence of a degree of a leverage effect. In order to resolve for the problem of "asymmetry" in volatility, the retained specifications point to the importance of stocks reactions in response to news. Effects of shocks on volatility highlight also the need to study the "long term" behavior of conditional variance of stocks returns and articulate the presence of long memory and dependence of time series in the long run. We note that the integrated fractional autoregressive model allows for representing time series that show long-term conditional variance thanks to fractional integration parameters. In order to stop at the dynamics that manage time series, a comparative study of the results of the different models will allow for better understanding volatility structure over the Tunisia stock market, with the aim of accurately predicting fluctuation risks.
\end{abstract}

Keywords: Volatility, Asymmetry, Clustering, Stylized Facts, Leverage Effect

\section{Introduction}

Financial markets have known over the last years important erratic movements characterized by a strong uncertainty about stocks returns and financial situation of firms. Stocks variability and the important deviation from fundamentals put an emphasis on the need to study in detail the notion of risk, a relevant concept to market variation.

The rapid development of financial instruments and liberalization of international capital movements came along a substantial increase in Trading. This trend has strongly exposed investors and financial institutions to market risk, i.e. to losses due to variations in stock prices.

Risk measurement has developed since the 1990s and remains a genuine utopian topic for finance researchers. Traditionally, the terms associated to risk articulate statistical uncertainty of earnings whose canonical measure consists in observing the effect of past information on the frequency of possible stocks variations and in assuming that these frequencies are the real mechanism of stocks evolution and that they are constant over time. The simplicity of this approach denigrates financial theory. Measuring risk through standard deviation of observations indicates, through its historic analysis, that this measure was not developed to take into account large fluctuations. Observing financial disorder and stocks fluctuations highlight indeed another type of risk, that which relates to perceiving volatility as a constant parameter, though it is in itself a volatile parameter.

Definition of volatility has exceeded the simple resemblance to variability of earnings and acquired a dominant position in the control process of big firms and decision-making of market operators. Researchers have extensively invested efforts in view of answering the following question: what is volatility? Understanding origins of volatility and analysing its variations may lead to a better perception of reality and control of market risk.

Over a century, research has abandoned the deterministic approach of Laplace-Gauss and ushered in a new forecasting way by inventing methods that are more adapted to describing reality and by integrating market uncertainty. Financial theory indicates that measurement of risk through Gaussian modeling is de facto inappropriate. Gaussian modeling of returns, on which traditional theory is founded and whose inadequacy results from the "central limit 
theorem", assumes that all random phenomena caused by a big number of independent small causes have the normal distribution as its probability law. An assumption that highlights the fact that reality represents the extreme opposite of a Gaussian universe. Description of empirical data needs then a mathematical modeling that is as faithful as possible to the observed behaviour.

The first models of time variations of fluctuations, treated under the "barbaric" term heteroscedasticity, refer to an autoregressive scheme in which we allow conditional standard deviation to evolve endogenously. The pioneering work [1] [2] [3] provides an adequate framework for the explicit modeling of price dynamics of large variations. A synthetic portion of the literature on the subject was provided notably by [4] [5] These studies indicate that autoregressive conditional volatility allows for extracting the portion anticipated ex- ante of historical volatility based on the possible different specifications of conditional variance behaviour. Without entering into a detailed discussion of dynamics, we specify that we target fluctuations of fluctuations scale, as opposed to historical variance which is necessarily the ex-post representation of past returns fluctuations.

The aim of this paper is to measure price fluctuations risk and to define volatility characteristics in the Tunisian stock market. The focus of our study is twofold. First, the concept of risk measure addresses the problem of modeling in terms of the degree of adapting statistics applicable to reality. We assume that the pricing model determines the use of predictions and is the source of uncertainty. Consensus about modeling techniques has become in recent years one of the most important issues of market risk management. Second, studying volatility characteristics is like focusing on the different stylized facts that exist in the market. Then, this paper is structured as follows. The second section focuses on the characteristics of time series, while the third section presents the models that are likely to define market fluctuations in terms of persistence, asymmetry and long memory. Finally, the fourth section presents a comparative analysis of the different obtained results.

\section{The Study of Volatility}

In this paper, we insist on the importance of volatility as a determinant of stock market fluctuations, inherent to understanding financial markets behavior. Often the central concern of those who seek financial security is to consider the best way to protect themselves against excessive financial markets volatility. Several authors argue that volatility is the raw material of market activities; decreasing it shall in no way be the sole objective of financial markets regulators alone.

In our study, we treat volatility through its property of assessing market fluctuations, in the sense of "writing up an equation" of reality from applied statistics. Indeed, the change in price of a financial asset in a future period is uncertain and should be able to translate the anticipations of fluctuations.

\subsection{Methodology}

In this study, we examine volatility over a significant period of the Tunis Stock Exchange, which runs from $1 / 1 / 1999$ to $15 / 06 / 2012$. This latter period represents a time interval that includes several episodes of market fluctuations. The data used are daily frequencies.

The used Tunindex is a synthetic index of the Tunis Stock Exchange. Published since April 1st 1998 with a 1000-base as of December 31st, the index is open to valid values through their common shares excluding investment companies and securities whose trading period is at least 6 months. The value of the index is given by the arithmetic mean of stocks prices compared to the index as weighted by market capitalization. The rate used to determine this index is the closing price.

The graphical analysis of fluctuations is based on a careful study of time series and highlights patterns in the evolution and dynamics of market fluctuations. Figure 1 shows the evolution of Tunindex returns in daily frequencies. In this figure, large movements of returns are often followed by large movements and small movements of returns are followed by small movements. This latter trend is known as "volatility clustering": a type of returns' heteroscedasticity that the Tunindex seems to display. The returns series seems stationary and exhibits dependence over time. Observing returns fluctuations of the market index highlights the following trends:

- Fluctuations in the series are variable in time and downward trends are much more frequent than upward trends.

- Dating trends shows periods of persistent shocks of any sign, but there is no recurrence of instability. The chronic movements have fairly regular long term aspect.

- The general trend of returns is also regular. There is an absence of a cyclic component. The series seems stationary, an intuition that can be supported by analyzing the returns series' correlation matrix as well as the usual stationarity tests.

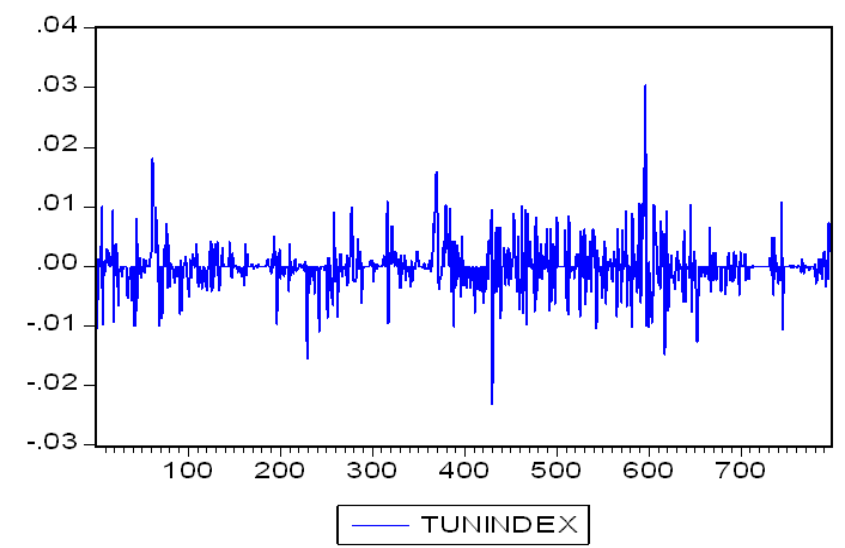

Figure 1. Tunindex Returns 


\subsection{Descriptive Statistics}

The study of descriptive statistics of the index's stock returns relates to analysing stationarity, autocorrelation, time series normality and heteroscedasticity of residuals. Our aim is to highlight the specificities of the Tunisian stock market through these various estimations and to deduce volatility characteristics.

To investigate stationarity, we should run first a detailed analysis of autocorrelation of financial time series. Autocorrelation functions of time series $\varphi_{\mathrm{k}}$ in our sample allow for measuring correlation of each returns' time series with itself lagged by $\mathrm{k}$ periods. Observing the correlation matrix of the Tunindex returns series, we find that Ljung and Box's $Q$ statistic is greater than $\chi^{2}$ at 12 degrees of freedom. This latter result enables us to reject the null hypothesis of $\varphi_{k}$ coefficients. Tunindex returns series therefore does not qualify as white noise.

Table 1. Autocorrelation of Tunindex returns

\begin{tabular}{|c|c|c|c|c|c|c|c|c|}
\hline $\mathbf{K}$ & 1 & & 2 & & 3 & & 20 & \\
\hline & $\varphi$ & $\mathbf{Q}$ & $\varphi$ & $\mathbf{Q}$ & $\varphi$ & $\mathbf{Q}$ & $\mathbf{Q}$ & $\varphi$ \\
\hline Tun & 0.11 & 9.43 & 0.12 & 19.76 & 0.17 & 42.32 & 39.34 & 0.09 \\
\hline
\end{tabular}

K: order autocorrelation, $\varphi$ : autocorrelation coefficient, Q: Ljung-Box statistic

Dickey and Fuller's augmented stationarity tests enable us to consider the possibility of the presence of errors autocorrelation. The tests check the stationarity of the series and determine the number of $\rho$ lags through Akaike or Schwartz criteria. Phillips and Perron tests allow us to take into account the presence of heteroscedastic errors, using the calculation of the long-term variance of the estimated residuals; the obtained PP statistic is compared to Mac Kinnon's table of critical values and used to detect the possible presence of a unit root.

The study of descriptive statistics of stock returns series allow for highlighting the specificities of the Tunisian stock market.

Table 2. DF Stationarity tests

\begin{tabular}{l|ll}
\hline Model (1) & -4.22401 & -1.940973 \\
Model (2) & -4.99588 & -2.890000 \\
& & \\
Model (3) & -4.14328 & -2.678512 \\
\hline
\end{tabular}

Model (1) without constant and without trend. Model (2) with constant and without trend. Model (3) with constant and trend. The third column represents the critical values at $5 \%$. The third column shows the critical values
Table 3: Results of PP stationarity tests

\begin{tabular}{l|ll}
\hline Model (4) & -9.40132 & -1.940969 \\
Model (5) & -59.39499 & -2.862665 \\
Model (6) & -59.66249 & -3.411871 \\
\hline
\end{tabular}

Model (4) without constant and without trend. Model (5) with constant and without trend. Model (6) with constant and trend. The third column represents the critical values at 5\%. The third column shows the critical values.

We note that for the unit root test, the result is the same when we applied the different models defined as the starting movements generating processes. The conclusions we reached are the same and confirm the rejection of the hypothesis of the presence of unit root; Tunindex series is stationary.

On the other hand, White's test allows us to study heteroscedasticity of residuals and identify any significant relationship that may exist between squared residual and one or more independent variables, simple or squared, in the regression equation:

$$
e_{t}^{2}=a_{1} x_{1 t}+b_{1} x_{1 t}^{2}+a_{2} x_{2 t}+b_{2} x_{2 t}^{2}+\ldots .+a_{k} x_{k t}+b_{k} x_{k t}^{2}+a_{0}+v_{t}(1)
$$

The results of White's heteroscedasticity test allow us to accept the heteroscedasticity hypothesis insofar as all regression coefficients are significantly different from zero.

Table 4. Results of the White's heteroscedasticity test

\begin{tabular}{ccc}
\hline & $\mathrm{TR}^{2}$ & $\mathrm{Q}$ \\
\hline 1 & 1.28977 & 0.256089 \\
2 & 1.30464 & 0.520834 \\
3 & 1.30310 & 0.728397 \\
4 & 1.46565 & 0.832728 \\
5 & 1.48927 & 0.914305 \\
\hline
\end{tabular}

We note that the rejection of homoscedasticity may be due to the presence of ARCH effect that is frequently encountered in financial time series, which assumes the presence of conditional heteroscedasticity. The difficulty of implementing this assumption is overcome by running another heteroscedasticity test, which is the ARCH test. This test allows us to calculate the model's squared residuals and proceed to an autoregressive regression of the series on $p$ lags, where only significant lags are kept. The Lagrange multiplier statistic LM compared with $\chi^{2}$ with $p$ degrees of freedom at the fixed threshold $(\alpha=0.05)$ allows us to conclude that the Tunindex process follows an ARCH (p) process.

Table 5. Study of the ARCH effect

\begin{tabular}{c|rcc}
\hline F-Statistic: & 1.289361 & Prob. F(1,2190) & 0.256290 \\
& 1.289779 & Prob. Chi-deux (1) & 0.256089 \\
\hline
\end{tabular}


Table 6. Study of normality

\begin{tabular}{ll}
\hline Statistics & Return \\
\hline Moyenne (\%) & 0.0463 \\
Déviation Standard (\%) & 0.351 \\
Skewness (S) & 0.864 \\
Kurtosis (K) & $(0.613)$ \\
Jarque-Bera & 98.76 \\
\hline
\end{tabular}

Given the evidence of non normality in emerging market returns presented in [6]. We test the normality of financial time series over the Tunis Stock Exchange. The normality hypothesis assumes that deviations of price from the normal are extremely rare and the probability of sudden stock market fluctuations is very limited. Specifically, for the case of a Gaussian variable changes are typically of the order of $\sigma$; the value of such a variable does not deviate from the average by more than $2 \sigma$ only in $5 \%$ of cases and its cumulatives of an order greater than 2 are also all assumed zero. To test the normality hypothesis, our study uses the Jarque and Bera statistic. Table 6 shows that the value of the JB statistic is greater than the critical value for all the stocks of our sample. The normality of distributions hypothesis is rejected at the $5 \%$ level. The third quartile of the distribution of returns is also high. The fourth which is greater than 3 indicates that the distribution of the Tunindex returns is leptokurtic. The normality hypothesis is definitively rejected.

\section{Models of Volatility}

For several years, the evolution of financial time series has been explained by simple linear ARMA-type models. These models have the advantage of being easily implemented to perform forecasts at any horizon. But this implementation advantage is their major limitation since their linear representation affects the formulation of the types of dynamics observed. Indeed, the dynamics of financial time series are

very complex and need appropriate regression models. Moreover, in light of the obtained results, the independence of returns series and the random walk hypotheses are strongly rejected, suggesting the presence of nonlinearity in the dynamics of returns series. Our study belongs to a classes of models introduced in 1982.[7][8] These models started with the following observation: the usual econometric tests conducted on time series use ARMA-type models leaving some data poorly explained, hence the need for a formulation of volatility that is more adapted to reality.

\subsection{Hetroscedastic Modeling of Returns}

We look for analyzing the volatility behaviour of our series with heteroscedastic errors. Our first finding is that volatility of the series is not constant. Non constant volatility shows that an ARCH-type process is well suited to model this variable.

$$
y_{t}=a_{0}+\sum_{i=1}^{j} a_{i} y_{t-i}+\varepsilon_{t}
$$

Non constant volatility is written by:

$$
\sigma_{t}^{2}=\alpha_{0}+\sum_{i=1}^{p} \alpha_{i} \varepsilon_{t-i}^{2}
$$

The lag order is identified by the synthesis of the characteristics of the different simple and partial correlations of the series that are a mixture of pure AR and MA processes. The results noted above indicate that the probabilities associated with the $\mathrm{TR}^{2}$ statistics are very close to zero. To account for this heteroscedasticity effect, Table 7 reports estimates of the variance equations performed through the GARCH $(1,1)$ model. It reports the log-likelihood, the estimated parameters, and the associated Akaike statistics.

We find that the estimated coefficients satisfy the positivity constraint and are significantly different from zero. The estimation results of the GARCH (1.1) model are based on a specification that provides a moving average process of a $\mathrm{p}$ order $(\mathrm{p}=1)$.

$$
\begin{aligned}
& r_{t}=\mu+\xi_{t}+b \xi_{t-1} \\
& \sigma_{t}^{2}=\omega+\alpha \xi_{t-1}^{2}+\beta \sigma_{t-1}^{2}
\end{aligned}
$$

Table 7 presents the estimates of the model; the term $(\alpha$ $+\beta$ ) measures the degree of persistence of conditional variance. The hypothesis of a strong persistence is generally accepted in major stock markets for high-frequency data and the literature succinctly reported strong evidence. Volatility persistence now tends to decrease when data frequency decreases. In the case of our study, the data are daily frequency, then volatility persistence phenomenon may be observed. We note that the sum of the coefficients $(\alpha+\beta)$ reflects stationarity of observations of conditional variance. Table 7 presents the main results of the estimation of the GARCH model. We notice that the value of $(\alpha+\beta)$ is less than 1 . Volatility persistence is accepted.

The likelihood ratio test to jointly nullify the coefficients $\alpha$ and $\beta$ clearly shows that volatility dynamics can be properly traced by the GARCH approach. The autoregressive coefficient of conditional volatility reached high values. The introduction of lagged values at the level of the variance equation significantly improves the results. The GARCH model also accounts for the phenomenon of volatility clustering where large (small) changes in volatility are usually followed by large (small) changes, which shows that when returns move strongly in one direction or the other, the investor increases the estimated future volatility to the extent that he predicts this parameter depending on the expected volatility and on the unpredictable shocks. The moving average component has positive signs for most stocks. This sign is attributed to non-synchronous trading in the market. 
The study of long-term dependencies in conditional volatility process is conducted through a graphical study of the partial auto-correlation functions of the index returns and of the functions of partial autocorrelations of first differences market returns. The figures indicate that the two observed autocorrelations show a clear decrease starting from the first lag. These autocorrelations are also significantly different from zero in the case of market returns and are significantly cancelled for the first difference returns. This allows us to deduce the non-permanent effect of shocks on market returns volatility.

Table 7. Results of the heteroscedastic regression model

\begin{tabular}{ccc}
\hline coefficient & GARCH(1.1) & P-value \\
\hline B0 & 0.000 & 0.03725 \\
B1 & 0.02364 & 0.022533 \\
B2 & 0.02423 & 0.021951 \\
B3 & 0.07224 & 0.01969 \\
B4 & 0.03018 & 0.01500 \\
B5 & 0.02828 & 0.04556 \\
B6 & 0.009194 & 0.01974 \\
B7 & 0.02122 & 0.00598 \\
$\boldsymbol{\alpha}$ & 0.22533 & - \\
$\boldsymbol{\beta}$ & 0.72089 & - \\
$\boldsymbol{\alpha}+\boldsymbol{\beta}$ & 0.9404 & - \\
AKAIKE & 1482 & - \\
BIC & 1356 & - \\
In L & 2243 & - \\
\hline
\end{tabular}

$\ln \mathrm{L}$ is the $\log$ likelihood of the model function, the value of $(\alpha+\beta)$ indicates the stationarity of the regression process and is an indicator of the degree of persistence in volatility

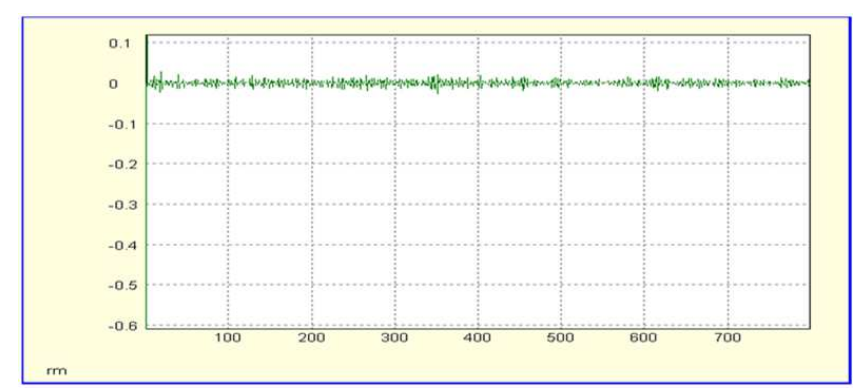

Figure 2. The partial autocorrelation function of TUNINDEX returns

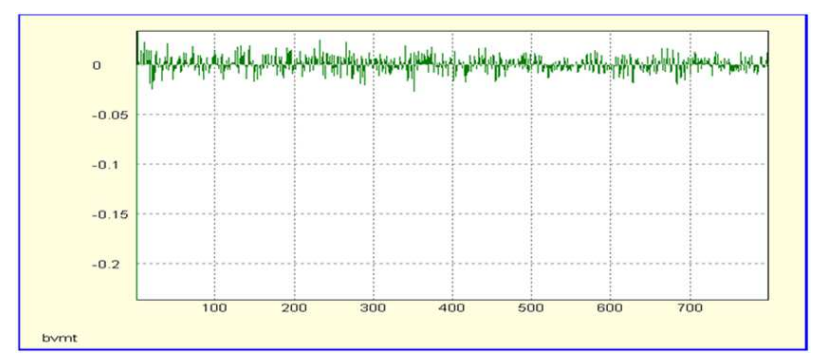

Figure 3 . The partial autocorrelation function yields TUNINDEX in first differences

\subsection{Shocs Asymmetry Effects on Volatility}

In this section, we examine volatility asymmetry in the Tunis stock market and negative correlation between returns and conditional volatility using leptokurtic effects heteroscedastic models.

\subsubsection{Asymmetry Test}

Specification [9] allowed us to detect volatility asymmetry. The aim of our study is to show that shocks differently affect volatility of the Tunisian financial market. Asymmetry manifests itself when volatility is high following a negative shock on conditional returns than following a positive shock. These tests use these four specific regressions:

$$
\begin{gathered}
\hat{z}_{t}^{2}=a_{1}+b_{1} S_{t-1}^{-}+e_{t} \\
\hat{z}_{t}^{2}=a_{2}+b_{2} S_{t-1}^{-} \hat{\xi}_{t-1}+e_{t} \\
\hat{z}_{t}^{2}=a_{3}+b_{3} S_{t-1}^{+} \hat{\xi}_{t-1}+e_{t} \\
\hat{z}_{t}^{2}=a_{4}+\beta_{1} S_{t-1}^{-}+\beta_{2} S_{t-1}^{+} \hat{\xi}_{t-1}+\beta_{3} S_{t-1}^{+} \hat{\xi}_{t-1}+e_{t}
\end{gathered}
$$

$a_{i,} \beta_{i}$ and $b_{i}$ are constant parameters

$$
S_{t-1}^{-} \text {is equal to } 1 \text { if } \hat{\xi}_{t-1 t} \prec 0
$$

and 0 otherwise.

$$
S_{t-1}^{+}=1-S_{t-1}^{-}
$$

\section{$e_{t}$ is the regression residual}

These diagnostic tests are run on raw data using the Student statistic. Several specifications are examined. The parameter $b_{1}$ indicates the presence of a biais of negative shocks on volatility (sign biais test). The parameter $b_{2}$ indicates whether biais of a negative return affects volatility (negative size biais test). The parameter $b_{3}$ indicates whether biais of a positive return affects volatility (positive size biais test). The joint test may be run as in equation 9. This test is run through the Lagrange multiplier $\xi=\mathrm{TR}^{2}$ where $\mathrm{T}$ is number of observations and $\mathrm{R}^{2}$ is the multiple correlation coefficient between the three variables. We emphasize that in case of asymmetry $\xi$ follows $\chi^{2}$ with 3 degrees of freedom.

Analysis of the statistical properties of returns showed that the normality hypothesis should be rejected because of asymmetry and kurtosis excess. Table 8 reports the main results. We notice that asymmetry tests are strongly significant. The $b_{2}$ indicates that the presence of biais of $a$ negative shock on volatility. The $b_{3}$ coefficient shows that a positive shock affects as well volatility but less than a negative shock as the $b_{2}$ coefficient is systematically superior in absolute value to the $b_{3}$ coefficient. This difference in biais, which is due to an asymmetry effect, is an important characteristic of volatility of the Tunisian stock market. This latter feature should be taken into account by investors during their predictions of future movement of stocks 
Table 8. Asymmetry test

\begin{tabular}{llllllll}
\hline \multirow{2}{*}{ Index } & \multirow{2}{*}{ LB (24) } & LB2 (24) & \multicolumn{2}{c}{ Asymmetry Test } & \multicolumn{2}{c}{ Test } & SCH \\
& & & b1 & b2 & b3 & Test \\
\multirow{2}{*}{ Tunindex } & 21.941 & 14.399 & 0.135 & 0.004 & -0.054 & $12 \mathrm{a}$ & $\mathbf{- 5 . 9 3}$ \\
& $(0.583)$ & $(0.810)$ & $(2.23)$ & $(1.054)$ & $(-1.2)$ & & \\
\hline
\end{tabular}

\subsubsection{Modeling}

The GARCH model does not account for volatility asymmetry. To overcome this problem, two specifications are used to better study volatility:

The EGARCH model [3] is defined by:

$$
\operatorname{Ln}\left(\sigma_{t}^{2}\right)=w+\beta \ln \left(\sigma_{t-1}^{2}\right)+\alpha\left|\frac{\xi_{t-1}}{\sigma_{t-1}}\right|+\gamma \frac{\xi_{t-1}}{\sigma_{t-1}}(10)
$$

Volatility is always positive, regardless of the values taken by the equation parameters. The process is stationary if the constraint $\beta<1$ is satisfied.

The TGARCH model [4] is represented as:

$$
\sigma_{t}^{2}=w+\alpha \xi_{t-1}^{2}+\gamma \xi_{t-1}^{2} d_{t-1}+\beta \sigma_{t-1}^{2}
$$

$d=1$ si $\xi_{t}<0$ and $d=0$ otherwise.

At the level of these two formulations, $\alpha$ represents the effect of a shock on returns and $\gamma$ is asymmetry effect manifested by a more virulent additional impact when the shock is negative than when it is positive.

For the EGARCH model, the positive effect of a shock takes the form of $(\alpha+\gamma)$. The negative impact is $(-\alpha+\gamma)$. The impact of shocks on returns is asymmetric if $\gamma \neq 0$.

For the TGARCH model, good news affect $\alpha$, while bad news affect $\beta$. The impact of shocks on volatility is also considered asymmetric when $\gamma \neq 0$. Asymmetry degrees are not directly compared from one model to another because they do not focus on the same endogenous variable. However, they allow us to compare the asymmetries of the different stocks, in that we can define and reconcile hierarchies deduced from the different approaches (from the strongest asymmetry degree to the weakest).

Table 9 reports the main synthetic statistical parameters of our study (log-likelihood, Ljunk-Box statistic). To compare the different asymmetric GARCH specifications, Schwartz criterion defined by $S=-21 / n+k \log n / n$ is taken into account (where $\mathrm{k}$ : number of estimated parameters $\mathrm{n}$ : number of observations and 1: log-likelihood value). The lower the value of the test is, the better the fit. The likelihood test allows for determining the distribution that best fits the data.

For the EGARCH specification, there is asymmetry. The TUNINDEX shows sensitivity to returns shocks. The value of $\gamma$ is 0.883 .

For the TGARCH model, presence of asymmetry hypothesis is also confirmed. We conclude that in the
Tunisian stock market, negative shocks generate greater volatility than positive shocks.

\begin{tabular}{|c|c|c|c|c|}
\hline Coefficient & $\begin{array}{l}\text { EGARCH } \\
\text { (1.1) }\end{array}$ & $\mathbf{P}$ & $\begin{array}{l}\text { TGARCH } \\
(1.1)\end{array}$ & $\mathbf{P}$ \\
\hline B0 & 0.0001 & 0.0165 & 0.0003 & 0.0376 \\
\hline B1 & 0.02364 & 0.0456 & 0.0245 & 0.03864 \\
\hline B2 & 0.02521 & 0.02654 & 0.0263 & 0.02167 \\
\hline B3 & 0.08522 & 0.01899 & 0.08204 & 0.01234 \\
\hline B4 & 0.03976 & 0.01323 & 0.03401 & 0.0564 \\
\hline B5 & 0.03283 & 0.04786 & 0.03654 & 0.03432 \\
\hline B6 & 0.00987 & 0.01364 & 0.0123 & 0.019886 \\
\hline B7 & 0.03122 & 0.04216 & 0.0345 & 0.00769 \\
\hline$\alpha$ & 0.04968 & - & 0.2355 & - \\
\hline$\beta$ & 0.17774 & - & 0.81259 & - \\
\hline$\delta$ & 0.8831 & - & 0.03274 & - \\
\hline AKAIKE & 1437 & - & 1259 & - \\
\hline SCHWARTZ & 1355 & - & 1202 & \\
\hline LB24 & 0.485 & & 0.074 & \\
\hline In $\mathbf{L}$ & 2258 & - & 2367 & \\
\hline
\end{tabular}

Table 9. Asymmetric modelling of volatility

\subsubsection{Persistence of Shocks to Volatility}

At this level, we focus on long-term behaviour of conditional variance of stock returns. It is customary to assume that the heteroscedastic modeling of financial time series implies a phenomenon of persistence of shocks to volatility, yet with persistence degree often overlooked. The results previously obtained by GARCH (1.1) highlight significant volatility persistence and presence of long memory, results which assume that fractionally integrated GARCH processes are best suited to describe reality. Our study is a similar attempt to model the concept of long memory. The FIGARCH (p ,d, q) [10] process allows us to provide a direct measure of this persistence through the fractional integration parameter (d). Akaike and Schwartz criteria will be then as comparison criteria of the different applied models.

To check for the effect of persistence of shocks on volatility, we first examine spectral density of returns series. Our reasoning is the following: if the returns have a long memory component, the spectrum will tend to the infinite if the angular frequency approaches zero. 


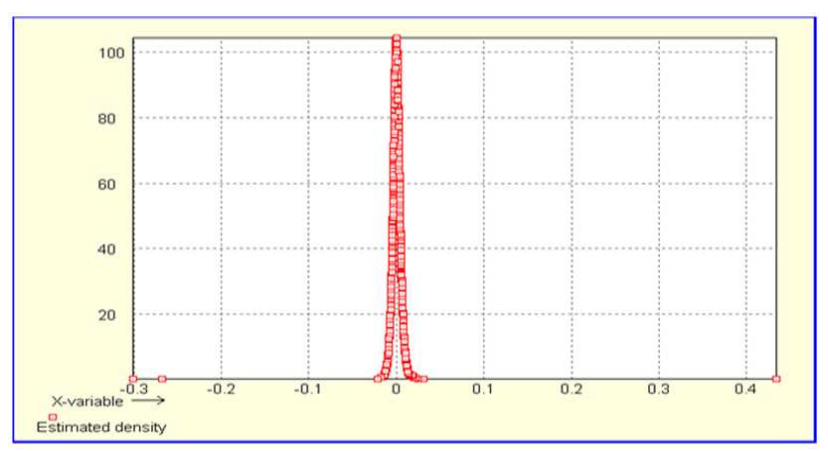

Figure 4. Tunindex spectral density (Spectral density Epanechnikov)

Figure 4 show that the series has a fairly low persistence, where spectral density does not approach the infinite around zero. However, this result is also not confirmed by the analysis of the series cumulative impulse-response function which represents the total impact of an innovation unit on the market's return process. Figure 5 shows a decline in the response function of the returns series which is due to innovation shock effect. This decline is followed by an increase representing the amortization of the innovation impact of the fractional differentiation parameter.

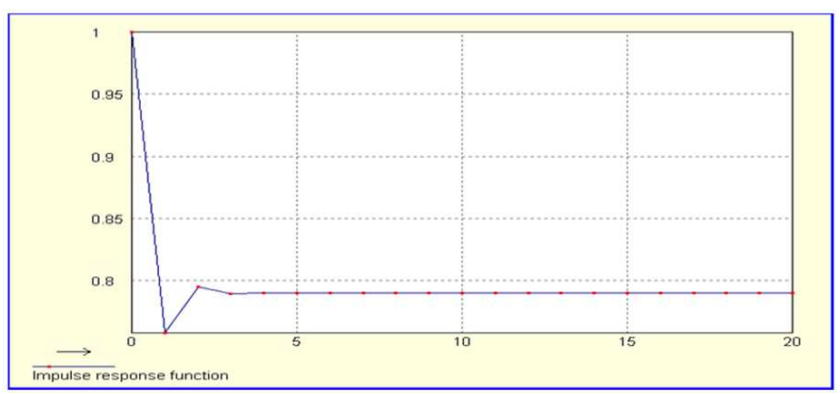

Figure 5. Response function of the market returns series

\subsubsection{Evaluation of Long Memory Parameter: Estimation Method Based on the G-P-H Procedure (1983)}

To attest for the hypothesis of shocks persistence effects on volatility, we apply the semi-parametric estimation method which will allow us to determine the coefficient (d); the fractional integration parameter in the conditional variance equation. The advantage of this parameter is that it allows for characterizing the long-term behaviour of the studied series. The short-term behaviour itself is considered through the autoregressive moving average parameters of ARFIMA $(p, d, q)$. This is explained by the fact that the impact of the parameter $d$ on remote observations decreases hyperbolically when the lag increases, while the effects of moving and autoregressive average parameters decrease exponentially.

The G-P-H procedure (1983) consists in estimating the differentiation parameter of the fractionally integrated ARIMA model where the parameter is not an integer and may take several values ranging between $-1 / 2$ and $1 / 2$. The estimation procedure is based on the regression: $\ln I\left(\lambda_{j}\right)=\operatorname{Ln} \frac{\sigma^{2} \Gamma_{\varepsilon}(0)}{2 \pi}-d \operatorname{Ln}\left[4 \sin ^{2}\left(\frac{\lambda_{j}}{2}\right)\right]+\ln \frac{\Gamma_{\varepsilon}\left(\lambda_{j}\right)}{\Gamma_{\varepsilon}(0)}+\operatorname{Ln} \frac{\mathrm{I}\left(\lambda_{j}\right)}{\Gamma_{x}\left(\lambda_{j}\right)}$

$$
\text { Where: } \quad I\left(\lambda_{j}\right)=\frac{1}{2 \pi T}\left|\sum_{j=1}^{T} x_{j} e^{i j \lambda}\right|^{2}
$$

$$
\lambda_{j}=\frac{2 \pi j}{T}
$$

The estimation results of the Tunindex are reported in Table 10. Different values were selected to examine the stability of the estimation.

Table 10. Parameter (d) estimation by the GPH method

\begin{tabular}{l|lll}
\hline Tunindex & 0.5 & 0.55 & 0.8 \\
$\mathbf{d}$ & 0.078912 & 0.09832 & 0.06958 \\
$\mathbf{t}_{\mathbf{d}}$ stat & 0.948769 & 1.07865 & 1.35432 \\
\hline
\end{tabular}

$t_{d}$ : The statistical value of Student

The application of the spectral method revealed the presence of long memory in market returns only when the fractional integration parameter is positive and significantly different from zero. However, the significance of the parameter $\mathrm{d}$ depends on the choice of the periodogram ordinates. The choice for different values is to examine the stability of estimators when the periodigram ordinates number varies.

In our study, we find that the coefficient (d) is significantly positive at the $10 \%, \mathrm{~d} \in[0 ; 0,5]$; it is 0.06 for $\mathrm{m}$ $=0.8$. This allows us to deduce the presence of volatility persistence. The financial returns series are not independent in time. The GPH method, applied to the market index, allows us to conclude that by means of an ARFIMA modeling, the series can be predicted in the long term

\subsubsection{Estimation Using Hurst Exponent}

The presence of long memory can be confirmed by estimating the parameter $\mathrm{H}$ (Hurst exponent, also called auto-similarity parameter) which refers to two statistics: traditional R/S and modified R/S.

Traditional $\mathrm{R} / \mathrm{S}$ statistic checks if the estimator of the Hurst exponent has a persistence phenomenon in order to classify the studied series according to their types of dependencies. Thus, if $\mathrm{H}=1 / 2$, then the process has no long-term dependence and is qualified as a short memory process (white noise ARMA).

If $1 / 2<\mathrm{H}<1$, then the process has a long memory with positive autocorrelations that decay slowly when number of lags increases.

Early studies of the R/S statistic showed that this latter is not robust in the presence of short memory and heteroscedasticity. To remedy this problem, Lo (1991) developed the rescaled $\mathrm{R} / \mathrm{S}$ range as an extension of the traditional R/S statistic. Referring to Lo (1991) statistics, we note the statistic $\mathrm{V}$,

defined by $V=\frac{\widetilde{Q}_{T}}{\sqrt{T}}$;, where $\widetilde{Q}_{T}$ is the rescaled R/S 
range, $\mathrm{T}$ is the number of observations.

Table 11 shows the values obtained from estimating R/S and rescaled $\mathrm{R} / \mathrm{S}$, the limit distribution denoted $\mathrm{V}$ of the rescaled $\mathrm{R} / \mathrm{S}$ will be compared to the critical value proposed by Lo (1991). Market returns series has a long-term dependence structure if and only if the value of the $\mathrm{V}$ statistic is greater than the critical value of Lo (1991). The two critical values provided by Lo (1991) are 1747 at the 5\% level and 1.62 at the $10 \%$ level.

Table 11. Estimation using Hurst exponent

\begin{tabular}{llll}
\hline Serie & R/S & \multicolumn{2}{l}{ Rescaled R/S } \\
& H & H & V \\
\hline Tunindex & 0.87852 & 0.63261 & 1.76332 \\
\hline
\end{tabular}

It is clear from the results that the values of $\mathrm{H}$ estimated by the traditional $\mathrm{R} / \mathrm{S}$ and rescaled $\mathrm{R} / \mathrm{S}$ are both significantly higher than 0.5 for the Tunindex. The hypothesis of the presence of long memory is retained.

We note that for the case of the rescaled $\mathrm{R} / \mathrm{S}$, the $\mathrm{R} / \mathrm{S}$ value estimated through the Hurst exponent is greater than that estimated by Lo method. This confirms the presence of time-series long-term dependence especially that the rescaled $\mathrm{R} / \mathrm{S}$ has a limit distribution robust in the presence of short memory.

In short, it is possible to set investment strategies on the
Tunisian stock market. Long-term memory seems to help explain volatility behaviour, with volatility persistence exacerbated by anchoring and risk aversion behaviour of Tunisian investors.

\subsubsection{Modeling}

We use the FIGARCH process to estimate volatility in the context where the fractional integration coefficient (d) was significant. Remember that the process FIGARCH (p, d, q) is defined by:

$$
\sigma_{t}^{2}=\alpha_{0}[1-\beta(L)]^{-1}+\lambda(L) \varepsilon_{t}^{2}
$$

with $\lambda(L)=\left\lfloor 1-[1-\beta(L)]^{-1} \phi(L)(1-L)^{d}\right\rfloor$

The FIGARCH process takes into account the dynamics of long-term volatility through the parameter (d). The short-term dynamics is modeled through the usual GARCH parameters.

We estimate the parameters of this process by the maximum likelihood method. The parameter $(\mathrm{d})$ is a real number which may take any values on the interval $[0,1]$. We notice that the persistence of a shock to conditional variance of a process FIGARCH (p, d, q) decreases in a hyperbolic manner when $0 \leq \mathrm{d}<1$. [8]. The results of estimating the model's parameters for the different series of our sample are presented in Table 12.

Table 12. Study of the fractionally integrated TUNINDEX behaviour

\begin{tabular}{|c|c|c|c|c|c|c|c|}
\hline & $\alpha_{0}$ & $\lambda(\mathbf{L})$ & d & $\mathbf{R}^{2}$ & $\mathbf{L L}$ & Aka & Sch \\
\hline $\begin{array}{l}\text { Tunindex } \\
\text { - }\end{array}$ & $\begin{array}{l}0.531 \\
(3.98)\end{array}$ & $\begin{array}{l}0.227 \\
(4.66)\end{array}$ & $\begin{array}{l}0.994 \\
(6.59)\end{array}$ & 0.09 & -42.92 & -7.78 & -7.76 \\
\hline
\end{tabular}

Values in parentheses represent statistics student, $\alpha 0$ is the autoregressive term, $\lambda(\mathrm{L})$ is the moving average value, $\mathrm{d}$ is the coefficient of fractional integration.

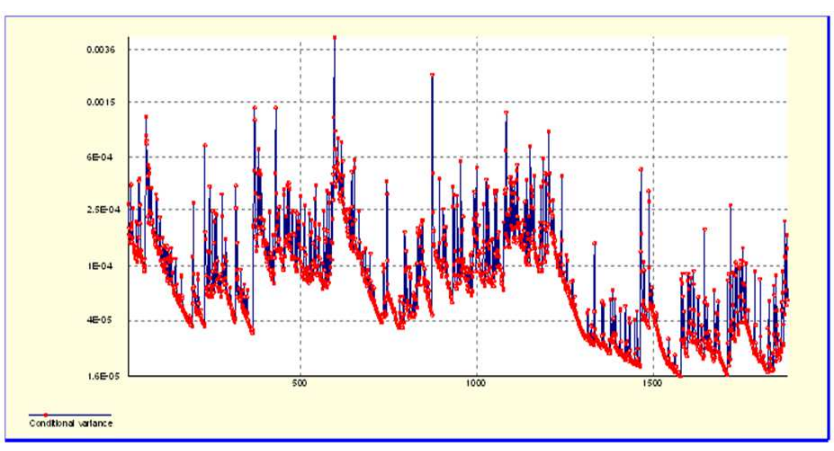

Figure 6. Representation of conditional volatility under the FIGARCH model

We notice that the fractionally integration parameter is as significantly different from zero for the Tunindex as for all the firms. These results suggest that the series returns exhibit long memory and induce volatility persistence to shocks of returns. It appears that the FIGARCH model is adequate to describe the dynamics of volatility on the Tunisian stock market. The presence of persistence predicts future volatility. We also notice that a shock causes an increase in conditional variance whose extent, indefinite in this study, can lead to an exaggerated volatility reaction.

Observing the evolution of the market index reported in Figure 6 allowed us to justify the empirical relevance of choosing the fractionally integrated process. Indeed, it seems clear that upward and downward shocks present a persistence component. The Tunindex time series is characterized by a significant heteroscedasticity which confirms the presence of long memory.

\section{The Comparative Study}

We conducted a comparative study of the results of conditional variance estimation of the different proposed volatility models. The choice of the returns-generating process can be done using various benchmarks. In particular, we traced log likelihood statistic and Akaike and Schwartz's information criterion to determine which distribution better fits the data. Thus, the lower the estimated values are, the better the fit is.

The study of the standardized residuals of the different estimation models revealed primarily that the standardized 
residuals series are like random variables, independently and identically distributed.

This result is confirmed by autocorrelation coefficients of standardized residuals and squared standardized residuals, which show that all studied models account for nonlinear dependencies of the returns series of the Tunis Stock Exchange.

Volatility structures in the context of good and bad news allow for tracing behaviour of prices throughout our study period. Figure 8 shows that this structure is different for each model. Volatility seems to be higher especially for the FIGARCH model, indicating that the fractional integration

\section{ARCH}

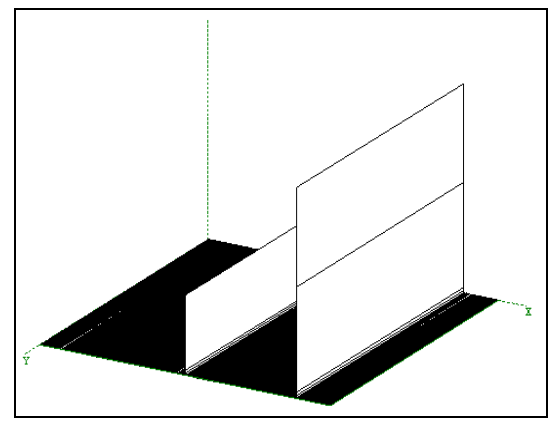

EGAR

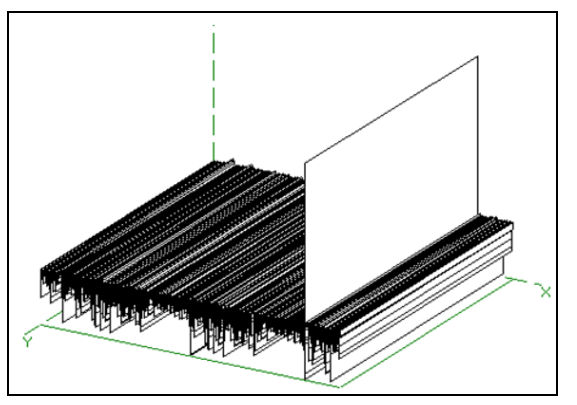

FIGARCH

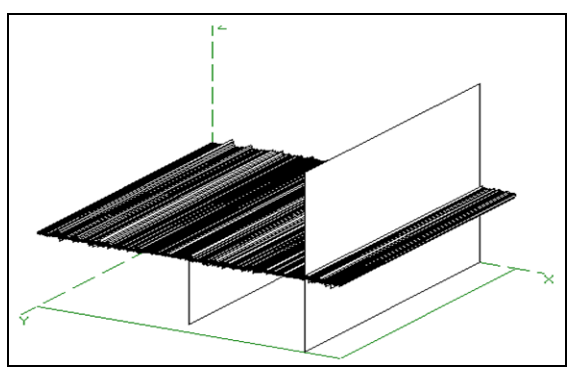

process displays significant peaks for higher frequencies of prices with some degree of persistence which represents a characteristic of a long memory process. Theoretical literature in finance [11] suggests that cross-sectional aggregation of news information arrival processes with different degrees of persistence could lead to long memory and fractional integrated model. The evidence in [12] find that fractional integrated model allows autocorrelation function to decay hyperbolically; financial markets go through periods of structural change producing volatility breaks and regime

\section{GARCH}
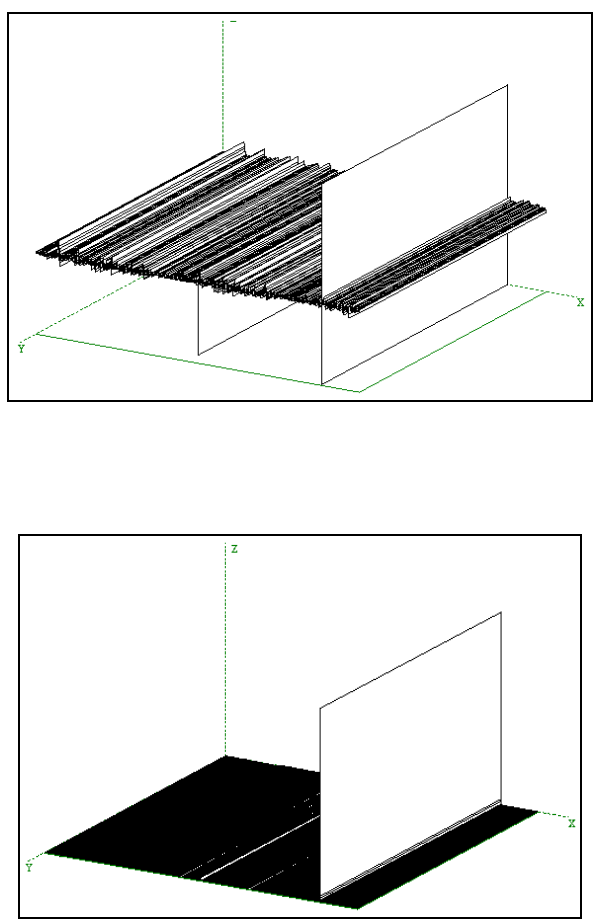

Figure 7. Volatility structures

shifts as consequence. Volatility is short memory between regime shifts, volatility break is the major cause of volatility long memory.

The structure of the EGARCH process shows the sensitivity of stocks both to good and bad news. We notice as well both upward and downward volatility fluctuation, reflecting the inclusion of the asymmetry hypothesis which characterizes the dynamics of financial time series. This representation is quite logical since the EGARCH process avoids the parameters positivity constraint unlike GARCH and $\mathrm{ARCH}$ processes whose structure is monotone. Several studies [13] find that asymmetric GARCH model capture 
asymmetric news impact and fat tailed distribution of central European stock market volatility. Other studies [14] find little evidence in support of the leverage hypothesis that explain asymmetric volatility and propose a model of conditional covariance that accommodates both the sign and magnitude of returns innovation.

The TGARCH model seems less fit to represent the dynamics of financial time series. We note that the TGARCH representation, which is a stepwise linear function where each step is associated with shocks of the same sign, is represented by a flat and uniform volatility structure reflecting a poor reaction of prices to the various shocks whatever their signs.

The results of comparing the performance of the risk estimation models are reported in Table 13. The estimated values show the superiority of the EGARCH model in representing Tunindex volatility; the benefit of using a nonparametric method against a standard GARCH $(1,1)$ is at 320.14 for the log-likelihood criterion and 2.07 and 2.02 respectively for Akaike and Schwartz criteria.

This suggests that the market shows a moderate but significant sensitivity to bad news and confirms the presence of leverage which is assumed to be responsible for the asymmetric impact of shocks on volatility.

The values of the log-likelihood criterion indicate on the other hand that the FIGARCH model is also able to describe behavior of financial time series that record long-term dependencies. Remember that long-term dynamics represented by the fractionally integrated parameter (d) have significant values. The TUNINDEX presents a long memory component. The ARFIMA representation is then neatly parsimonious to represent volatility. Earlier studies [15] report evidence of the presence of fractionally integrated behavior in the conditional variance of S\&P 500 returns and find that FIGARCH specification is better than GARCH $(p, q)$. Several studies [16] find that long memory in conditional variance and high volatility persistence tends to increase in crises periods.

Table 13. Comparisons Criteria of the regression models

\begin{tabular}{llll}
\hline Model & Log likelihood & Akaike & Schwartz \\
\hline ARCH (1) & 6853.214513 & -5.59119552 & -5.58171985 \\
GARCH(1,1) & 7565.115891 & -6.17152317 & -6.15967859 \\
E-GARCH & 7885.255587 & -8.24398754 & -8.17654298 \\
T-GARCH & 3013.343245 & -7.99965422 & -7.98453266 \\
FIGARCH & 7599.90494 & -6.20000404 & -8.18104631 \\
\hline
\end{tabular}

Presence of persistence effect is a limitation of an $\mathrm{ARCH}$ modelling as it is well known that an ARCH model is unable to entirely identify volatility persistence effect. Nevertheless, we note that our estimation was unable to reject the $\mathrm{ARCH}$ $(1,1)$ effect. The log-likelihood function is 6853.2 for the TUNINDEX.

In conclusion, we note that the most performing models for Tunisian market returns data are inevitably those that accommodate a leverage effect, the volatility of equity returns exhibits an asymmetric reaction to positive and negative shocks. Economic explanations suggested for this phenomenon are leverage and a volatility feedback effect [17] [18].

\section{Conclusion}

As risk-return arbitrage is the substance of finance, volatility has often been an essential parameter of portfolio management. Thus, accurate and reliable measures of predicting volatility are needed in econometrics.

In our study, we put an emphasis on the econometric difficulties in evaluating risk through traditional measures of volatility: (1) financial risk is a loss risk and is not a profit risk, a definition where events play symmetrical roles less conform to reality, (2) Gaussian hypothesis of modelling returns is never justified for large fluctuations. Distribution tales which manage frequencies of large movements are poorly described by the normal distribution law, (3) presence of important shapes of fluctuations introduces observations that lead to estimations not supported by volatility.

Our study highlighted models that allow predicting fluctuations of stocks. The ARCH-GARCH methodology reflects instability of volatility in time. The reference scheme is an autoregressive scheme in which we allow for the conditional standard deviation to evolve endogenously. We notice that it is about a specification that takes into account some stylized facts like persistence of financial returns and presence of a leverage effect. However, this specification includes loss of precious information, which a sign of past errors.

To solve for this problem, more sophisticated models were the object of our attention. For these models, an asymmetric response of volatility to shocks of returns of opposite signs may take place and an evaluation of the long-term behavior of prices in the presence of significant heteroscedasticity is de facto required.

Moreover, facing these many limitations of each specification, we notice that the choice of a volatility model is often antagonistic. Each particular financial specification depends on the characteristics of the integrated variable. Variability effects are less powerful, particularly for short time horizons and become observable later. This is a concept shows us an additional theoretical study of volatility is a priori necessary for a detailed description of prices variations.

\section{References}

[1] R. Engle (1982), "Autoregressive conditional heteroscedasticity with estimates of the variance", Econometrica, vol 50 (4), pp 987-1007

[2] T. Bollerslev (1986), "A conditionally heteroscedastic time series model of security prices and rates of return data", Review of economics and statistics, vol 59, pp 542-547 
[3] D. Nelson (1990), "ARCH models as diffusion approximations", Journal of econometrics vol 35, pp 7-38

[4] J.M. Zakoin (1994), "Threshold Heteroscedastic Models", Journal of Economic Dynamics and control, Vol 18, pp 931-955.

[5] R. Engle and G. Rivera (2006), "Semiparametric ARCH models", Journal of business and economic statistics, vol 9 (4), pp 345-359

[6] L Hentschell (1995), "All in the family: Nesting symmetric and asymmetric GARCH models", Journal of financial economics vol 39, pp 71-104.

[7] P. Hansen and A. Lunde (2005), "A Forecast comparaison of volatility models: does anything beat a GARCH (1.1)", Journal of Applied Econometrics; vol 20, pp 873-889.

[8] S. Ling and M. MacAleer (2000), "Testing GARCH versus EGARCH, Statistics and finance: An inference", Imperial college Press London, pp 226-242

[9] R. Engle and V. K. Ng (1993), "Measuring and testing the impact of News on volatility", Journal of Finance, vol 48, pp 1749-1778.

[10] T. Bollerslev and H.O Mikkeslen (1996), "Modeling and pricing memory in stock market volatility", Journal of Econometrics, vol 73, pp 151-184.
[11] B.Anderson and A. Lund (2002), An empirical investigation on Continuous time return equity models, Journal of Finance, vol 57, 1239-1284

[12] N.S. Hyung and C. Granger (2006), A source of long memory in volatility, working paper series

[13] P. G. Patev and N. k. kanaryan (2004) "Modelling and forcasting the volatility in central European stock market", working paper series

[14] W. G. Dean and R. W. Faff (2001), "Asymmetric covariance, volatility and the impact of news", working paper series

[15] G. Beakeart and G. Wu (2000), "Asymmetric volatility and Risk in Equity Markets”, Review of Financial Studies, vol 13, pp 1-42.

[16] T. Bollerslev and J. M. Wooldrige (1992), "Quasi Maximum likelihood estimation and Inference in Dynamic models with Time-Varying Covariance", Econometric reviews, vol 11(2), pp 143-172.2.

[17] G. Beakeart and G. Wu (2000), "Asymmetric volatility and Risk in Equity Markets", Review of Financial Studies, vol 13, pp $1-42$.

[18] K. Bea and R. Nelson (2004), "Why are stock returns and volatility negatively correlated?" Journal of financial economics, vol 24, pp 124- 151. 